\title{
A method for large scale introduction of wind power generation using PSO under frequency constraints
}

\author{
Yuya Maruno, Toshiki Takayama*, Shinichi Iwamoto \\ Waseda University, 3-4-1 Okubo Shinjuku-ku, Tokyo, 169-8555, Japan
}

\begin{abstract}
Recently, environmental problems have prompted the prevalence of renewable energy sources such as wind power. In addition, safety concerns about nuclear power plants in Japan have also increased the adoption of renewable energy technology. In this paper, we use a nonlinear load frequency control model to propose a method for optimal allocation and distribution of wind power to improve the amount of renewable energy in the power grid. Furthermore, when we introduce the wind power under system frequency constraints, we use particle swarm optimization (PSO) and frequency sensitivity to make this proposed method more realistic. Then, to verify the validity of the proposed method, we perform a numerical simulation using a 9-machine 20-bus system.
\end{abstract}

Keywords: Load frequency control, nonlinear model, particle swarm optimization, wind power, renewable energy

\section{Introduction}

We are currently faced with energy security problems such as the depletion of fossil energy resources as well as with environmental problems associated with greenhouse gas emissions [1], [2]. These concerns, along with the Fukushima Daiichi Nuclear Power Plant crisis caused by the Great East Japan Earthquake of March 2011, have led to increased calls for greater utilization of renewable energy sources in the power system. Especially, it is expected that wind power generations are installed in power systems because of reasons that the cost to install is relatively cheaper than other renewable energies.

However, the objective of load frequency control (LFC) is to maintain frequency quality by locally suppressing frequency deviations and tie-line power flow deviations within certain tolerances. Thus we focus on negative influences, such as frequency deviations caused by generator output variations [3].

With this background, we propose a method to increase the amount of wind power, under imposed frequency constraints $(f=0.2 \mathrm{~Hz})$, by optimally locating the wind power generators. Concretely, we use particle swarm optimization [4] (PSO), which is one of the metaheuristics, and use a sensitivity of system frequency (frequency sensitivity).

Next, instead of the conventional linear LFC model, we employ a nonlinear LFC model to calculate state variables in the system by solving the swing equations of individual generators.

To verify the validity of our proposed method, we carry out numerical simulations using a 9-machine 20-bus system [5].

\section{Wind Power Plant}

Wind power can affect LFC performance because of its variable output. However, wind power plant installation has increased rapidly in Japan for the following reasons.

- Wind provides clean energy.

* Manuscript received May 16, 2013; revised July 29, 2013.

Corresponding author. Tel.: +81 3-5286-3184; E-mail address: takayama.toshiki.pwrs@ gmail.com. 
- Wind is inexhaustible.

- Wind is a domestic resource.

- The cost to generate wind power is less than that for other renewable energies.

- Many wind power sources are expected to be able to connect to the power grid.

The future installed capacity of wind power is expected to increase. As shown in Table 1, high targets for wind power generation in 2020 and 2030 have been set by groups such as the Ministry of Economy, Trade and Industry, the New Energy and Industrial Technology Development Organization (NEDO), and the Japan Wind Power Association [6]; these goals illustrate the high expectations for wind power generation.

Table 1. Targets for installed wind power generation capacity in Japan

\begin{tabular}{c|c|c|}
\hline \multirow{2}{*}{} & \multicolumn{2}{|c}{$\begin{array}{c}\text { Wind power generation capacity } \\
\text { targets by year }\end{array}$} \\
\cline { 2 - 3 } & 2020 & 2030 \\
\hline METI & $5.0 \mathrm{GW}$ & $6.7 \mathrm{GW}$ \\
\hline NEDO & $10 \mathrm{GW}$ & $20 \mathrm{GW}$ \\
\hline JWPA & $8 \sim 12 \mathrm{GW}$ & $13 \sim 28 \mathrm{GW}$ \\
\hline
\end{tabular}

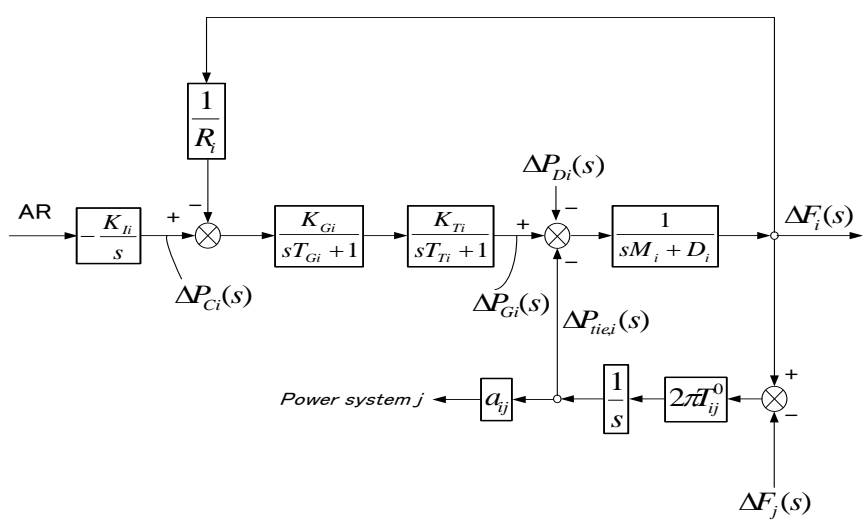

Fig. 2. Basic linear model of a power system (area $i)[8]$.

\section{LFC Model}

LFC simulation models [7] use a system model linearized around operating points of the power system, as shown in Fig. 2 [8].

However, in the linearized model, it is difficult to determine the voltage distribution and power flow conditions of the system, and their influence cannot be considered. Additionally, all generators in the system are assumed to be synchronized, resulting in an aggregated model where all the generators are integrated into one. In the analysis of the linearized model, the system has a tendency to stabilize even if a large disturbance occurs that makes generator synchronization unrealistic. Therefore, when adding a large amount of renewable energy to the system, some situations will arise that cannot be analyzed by the linearized model.

In this paper, we perform a nonlinear model analysis by solving the swing equation of an individual generator to calculate the state variables in the system. The equations of the generator used for the analysis is shown below [9]:

$$
\begin{aligned}
& \frac{d \delta_{i}}{d t}=\omega_{i}-2 \pi f_{0} \\
& \frac{d \omega_{i}}{d t}=\frac{\pi f}{H_{i}}\left(P_{m i}-P_{e i}\right)
\end{aligned}
$$


where $\omega$ is angular velocity, $P_{m}$ is mechanical power, $P_{e}$ is electric power, $H$ is the per-unit inertia constant, $f$ is frequency, and $i=1,2, \mathrm{~L}, n$ are the generator numbers.

We then introduce the governor shown in Fig. 3, where $R$ is speed regulation, $K$ is gain, and $T$ is a time constant.

The governor is represented by a first-order model. Fig. 3 shows how we attempt to cancel out frequency deviations by adding the control variable $\Delta P_{c}$ to the governor. The calculation method of $\Delta P_{c}$ is shown in Fig. 4.

In Fig. 4, area control error (ACE) is calculated to locally adjust the supply and demand balance. For the sake of simplicity, we assume that the signal $u$ passed from the controller is equally distributed to each generator.

In the analysis, (1) and (2) are solved simultaneously with the network equation to calculate the power flow conditions of the system.

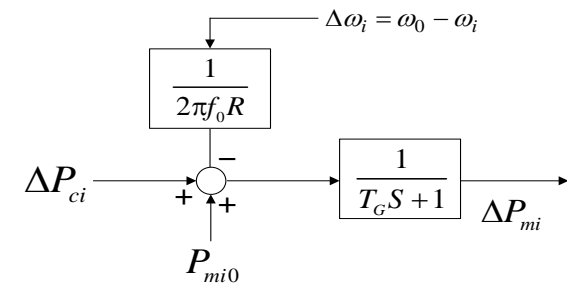

Fig. 3. Block diagram of the governor

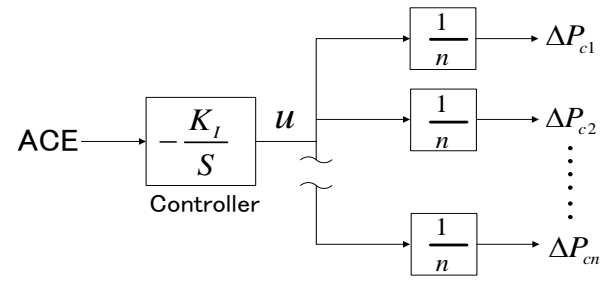

Fig. 4. Concept of the $\Delta P_{c}$ calculation

\section{Particle Swarm Optimization}

In this paper, we use PSO [4], which is one of the metaheuristics, to decide the optimally positioning and distributing wind power.

PSO is a stochastic search technique which was proposed by J. Kennedy and R. Eberhart in 1995. PSO algorithm imitates the behavior of a flock of birds, and each bird, here agent, search for good solutions using information of position and velocity. Although the algorithm uses only fundamental arithmetic operations, PSO has a good search performance. The movement of the each agent from one point to the next is determined with a velocity vector defined as (3). Each agent knows the best position from its own experience $\left(p_{\text {ibest }}\right)$ besides the best known position of whole flocks $\left(g_{\text {best }}\right)$. The next velocity vector is determined by $p_{\text {ibest }}, g_{\text {best }}$ and current velocity vector.

$$
v_{i}^{k+1}=\omega \cdot v_{i}^{k}+c_{1} \cdot \operatorname{rand}_{1} \cdot\left(p_{\text {ibest }}-x_{i}^{k}\right)+c_{2} \cdot \operatorname{rand}_{2} \cdot\left(g_{\text {best }}-x_{i}^{k}\right)
$$

where $v_{i}^{k}$ is a velocity vector ( $k$ : iteration count, $i$ : agent number); $x_{i}^{k}$ is the search point; $\omega$ is a weight of the current velocity vector; $c_{i}$ is a constant; rand is a uniform random number between 0 and 1 ; $p_{i b e s t}$ is the position of the best solution of each agent $i$; $\mathrm{g}_{\text {best }}$ is the position of the best solution among all agents.

$\omega$ (a weight of the first term) and $c_{i}$ (weights of the second and third term) are defined as follows. ${ }^{[4]}$

$$
\omega=\omega_{\max }-\frac{\omega_{\max }-\omega_{\min }}{\text { iter }} \times \text { iter }
$$

where $\omega_{\max }=0.9, \omega_{\min }=0.4$, iter is iteration count, and iter $_{\max }$ is maximum iteration count.

$$
c_{1}=c_{2}=2
$$

Each agent updates its search point by (6) until it reaches the maximum iteration count. 


$$
x_{i}^{k+1}=x_{i}^{k}+v_{i}^{k+1}
$$

\section{Proposed Method}

The objective of this paper is to increase the contribution of wind power to the system within the imposed frequency constraints; this section will explain the proposed method to accomplish this objective.

\subsection{A method for large scale introduction considering total amounts of wind powers (Method 1)}

For increasing the contribution of wind power to some area, we use PSO with an evaluation function shown in (7).

$$
\text { Evaluate } 1=\sum P w_{j}-\omega_{1} \cdot \text { penalty } 1-\omega_{2} \cdot \text { penalty } 2
$$

where $j$ is load bus number, $P w_{j}$ is the amounts of wind power introduced into the bus $j, \omega_{1}$ and $\omega_{2}$ are the weight of a penalty term.

Penalty 1 is the frequency constraints:

$$
\left\{\begin{array}{cc}
0 & \left|\Delta f_{\text {all }}\right|_{\max }<\Delta f_{\text {lim }} \\
1 & \text { otherwise }
\end{array}\right.
$$

Penalty 2 is the constraint of available introduction amounts:

$$
\begin{cases}0 & P w_{j} \leqq P w_{\max j} \\ 1 & \text { otherwise }\end{cases}
$$

$\left|\Delta f_{\text {all }}\right|_{\text {max }}$ is the maximum value of frequency deviations in the all generators, $\Delta f_{\text {lim }}$ is the upper limited of frequency deviations , $P w_{\operatorname{maxj}}$ is the maximum possible amounts of introduction.

In (7), the first term shows the total amount of wind powers. Then, we consider the second term for a penalty of frequency constraints and the third term for a penalty of the constraint of available introduction amounts. When we search for a good solution by using PSO, we choose a solution whose evaluation function value is higher.

\subsection{A method for large scale introduction considering total amounts of wind powers and frequency sensitivity (Method 2)}

In Method 1, we simply consider the total amount of the wind power. However, the frequency by each load bus is different in each case. In this paper, we propose a more realistic method by adding frequency sensitivity of each load bus to evaluation function, which is defined as Method 2.

\section{1) Frequency sensitivity}

When we install the same amount of wind power to each load bus, the effects on system frequency are different. Then, we define a new index which is calculated by (8). We use the 'frequency sensitivity' as the new index.

$$
m_{j}=\frac{\Delta\left|\Delta f_{\text {all }}\right|_{\max }}{\Delta P w_{j}}
$$

where $m_{j}$ is the frequency sensitivity, $\Delta P w_{j}$ is the variation of installed wind power, $\Delta\left|\Delta f_{\text {all }}\right|_{\max }$ is the variation of maximum of frequency deviations in the all generators

Frequency sensitivity shows the effect on system frequency against the amounts of wind power. The larger the value of frequency sensitivity is, the more the introduction of wind power effect on the system frequency. Therefore, when we consider the large scale introduction of wind power, we should install the wind power to the load bus which has a lower value of the frequency sensitivity to improve the amount of 
the wind power introduction.

\section{2) Evaluation function of Method 2}

A new evaluation function which includes frequency sensitivity is shown in (9) as follows:

Evaluate $2=\sum_{j} \frac{1}{m_{j}} P w_{j}-\omega_{1} \cdot$ penalty $1-\omega_{2} \cdot$ penalty 2

In the first term of (9), we multiply a reciprocal of the frequency sensitivity. By multiplying the reciprocal, the introduction to the load bus which has low frequency sensitivity gives better solution than the introduction to the load bus which has high frequency sensitivity. The second is the penalty of frequency constraints, and the third term is the penalty of the constraint of available introduction amounts.

When we search for a good solution by using PSO, we choose a solution whose evaluate function value is higher.

\section{Simulation}

We run the nonlinear LFC simulation to confirm the validity of the method proposed in Section V. Specifically, we apply the method to the 9-machine 20-bus system shown in Fig. 6 and verify that the increase in wind power contribution is greater than in the conventional.

\subsection{Simulation Conditions}

In the simulation, we aim at increasing the contribution of wind power to the power system in Area 1. The simulation conditions and assumptions are as follows:

- We assume the control scheme of each area to be tie-line bias control (TBC) and flat frequency control (FFC).ACE in area $\mathrm{j}$ is expressed by the following equations [8]:

$$
\begin{aligned}
& A C E_{1}=-\beta \Delta f_{1}-\Delta P_{\text {tie }} \\
& A C E_{2}=\Delta f_{4}
\end{aligned}
$$

In (10), $\Delta P_{\text {tie }}$ is the tie line power flow deviation and $\beta$ is the frequency bias value. The general expression of $\beta$ is the following:

$$
\beta=D+\frac{1}{R}
$$

Here $D$ is the damping coefficient of the power system representing the frequency characteristics of the load. Specifically, in this paper, We set $D=0$ in (12).

- For comparison, we consider a simple method. We assume that the simple method is to connect the same amount of renewable energy in every load bus.

- We assume the frequency deviation tolerance to be $\pm 0.2[\mathrm{~Hz}]$. We connect wind power sources until a generator output violates the frequency constraints.

- In PSO, we assume that the total number of chromosomes is 30 and the number of iteration is 50 . Then, we use the parameters of PSO shown in (4) and (5).

- We use $\omega_{1}=\omega_{2}=100$ as the weight of evaluation function.

- We assume that the maximum possible amount of introduction is $20 \%$ of the estimated value of each load bus.

- In the simulation, we consider the maximum wind power contribution; thus, we use step type disturbances that are assumed to be wind power. 


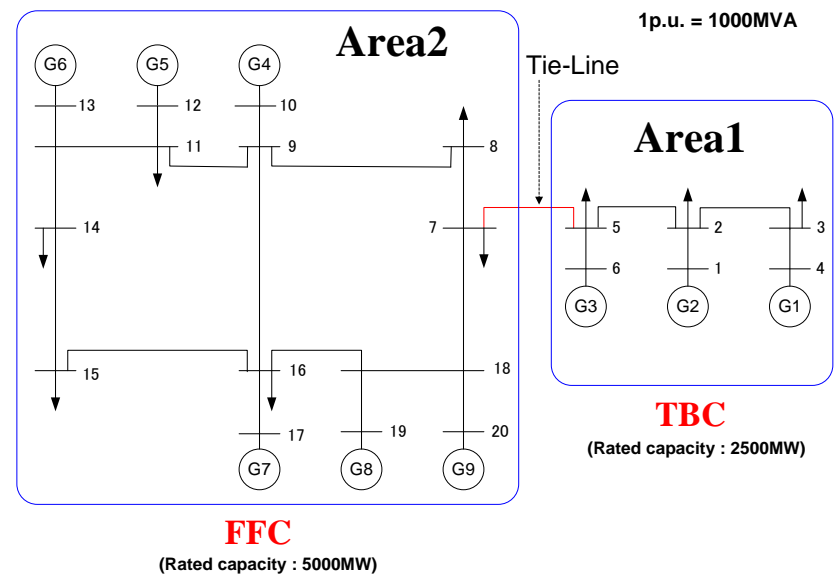

Fig. 6. 9-machine 20-bus system [5].

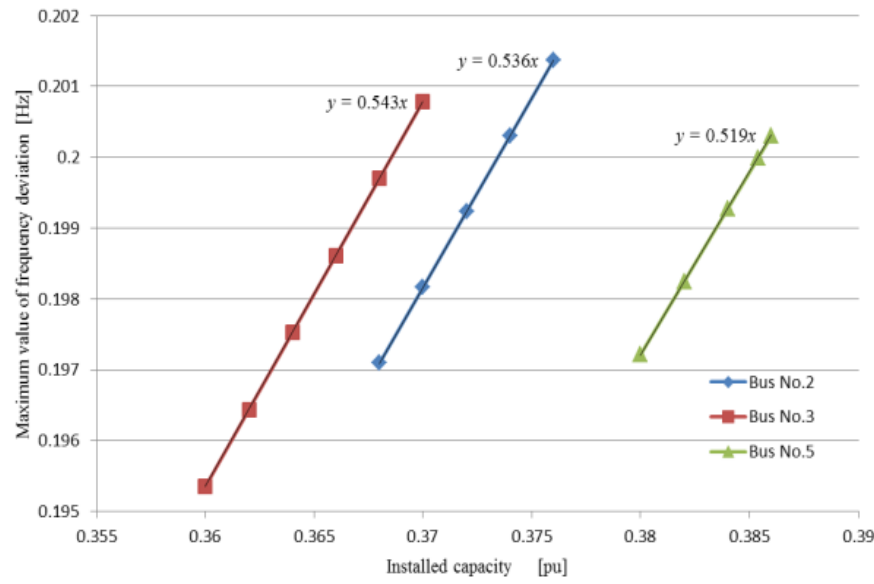

Fig. 7. The frequency sensitivity.

\subsection{Calculation of frequency sensitivity}

We calculate the frequency sensitivity which is used in Method 2. In bus No. No. 2, No. 3 and No. 5 which can be introduced wind power, we vary the amount of wind power introduction. Then, we show the variation of maximum frequency deviation in Fig. 7.

From Fig. 7, we got the linearity between installed capacity and maximum value of frequency deviation. Therefore, the slope of a line is frequency sensitivity $m_{j}$ of a load bus. In this simulation, we use these values in (13) as $m_{j}$.

$$
m_{2}=0.536, m_{3}=0.543, m_{5}=0.519
$$

\subsection{Result of the simulation}

We show the results of simulation in Table 3. This table shows the amounts of wind power introduction by using the simple method, Method 1, and Method 2.

Table 2. Simulation results

\begin{tabular}{cccccc}
\hline \hline Bus Number & Simple Method & Method 1 & Method 2 & Frequency sensitivity & Upper capacity \\
\hline 2 & 0.122 & 0.13 & 0.196 & 0.536 & 0.2 \\
3 & 0.122 & 0.187 & 0.1 & 0.543 & 0.2 \\
5 & 0.122 & 0.053 & 0.078 & 0.519 & 0.08 \\
Total & 0.366 & 0.37 & 0.374 & - & - \\
\hline \hline
\end{tabular}


From this result, we confirm that both of the proposed methods, Method 1 and Method 2, can improve the wind power contributions within the constraint of available introduction amounts. Furthermore, by using Method 2, we confirmed that the contribution of wind power to the system increased compared with Method1, because we preferentially introduced the wind power to the load buses which have low values of frequency sensitivities. Based on the above result, we confirmed the validity of the proposed method.

\section{Conclusions}

We have used a nonlinear LFC model that performs a more detailed analysis than the conventional linear LFC model. Then, we determined the optimal locations to connect the wind power and optimized the distribution of the added wind power by using PSO which is one of the metaheuristics.

Also, we have proposed a method for large scale wind power introduction considering system frequency constraints and the constraints of available introduction amount. Furthermore, we have proposed the method considering the frequency sensitivity which shows the effect on system frequency.

Finally, to confirm the validity of the proposed methods, we ran the nonlinear LFC simulation for a 9machine 20-bus system. From the results, we confirmed that both of the proposed methods improved the wind power contributions within the constraint of available introduction amount. Furthermore, by using Method 2, we confirmed that the contribution of wind power increased compared with Method 1, because we preferentially introduced the wind power to the load buses which have small frequency sensitivities.

\section{References}

[1] Bevrani H, Gosh A, Ledwich G. Renewable energy sources and frequency regulation: survey and new perspectives. IET Renewable Power Generation, 2010; 4(5):438-457.

[2] Arita M, Yokoyama A, Tada Y. Evaluation of battery system for frequency control in interconnected power system with a large penetration of wind power generation. In: Proc. of 2006 Int. Conference on Power System Technology, 2006:1-7.

[3] Wen T. Load frequency control: problems and solutions. In: Proc. of the 30th Chinese Control Conference, 2011:6281-6286.

[4] Kennedy J, Eberhart R. Particle Swarm Optimization. In: Proc. of IEEE International Conference on Neural Networks, 1995:1942-1948.

[5] Electric Joint Research. The Stability of power systems Vol. 34, No.5 (in Japanese)

[6] NEDO website. [Online]. Available: http://www.nedo.go.jp/

[7] Maruno Y, Iwamoto S. A consideration of load frequency control using non-linear models. Presented at: IEEJ National Convention, 2011 (in Japanese).

[8] Elgerd OI. Electric Energy System Theory: An Introduction. McGraw-Hill Book Company; 1982.

[9] Anderson PM, Fouad AA. Power System Control and Stability. Iowa State University Press; 1977:365-419. 